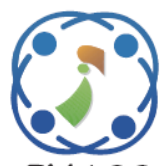

\title{
Reduction of Reporting Time for Throughput Enhancement in Cooperative Spectrum Sensing Based Cognitive Radio
}

\author{
Metta Venkata Satya Sairam $^{1^{*}} \quad$ Mupparaju Sivaparvathi $^{1}$ \\ ${ }^{I}$ Department of Electronics and Communication Engineering, G. V. P. College of Engineering (Autonomous), \\ Visakhapatnam, Andhra Pradesh, India. \\ * Corresponding author’s Email: sairammvs3@gmail.com
}

\begin{abstract}
Radio spectrum ( $3 \mathrm{KHz}$ to $300 \mathrm{GHz}$ ) is a limited natural resource and efficient utilization of the spectrum can be accomplished by cognitive radio (CR). In CR, spectrum sensing is one of the significant challenging issues. Cooperative spectrum sensing (CSS) based CR is a captivating solution to enhance spectrum sensing. In CSS, multiple secondary users (SUs) report their results to fusion center (FC) and final decision is taken on the status of the primary user (PU) based on the predetermined fusion rule. This cooperative decision in CSS based CR, improves the spectrum sensing performance. Nevertheless, the foremost drawback in CSS is low throughput due to reporting time introduced by the multiple SUs. We propose a novel method to reduce the reporting time so as to enhance the throughput performance for the same number of SUs in CSS based CR. In the proposed scheme $\mathrm{k}$ SUs, where $\mathrm{k}$ is even, are divided into two groups, each with $\mathrm{k} / 2$ users. Two groups report their sensing results to two FCs separately and final decision is taken by the third FC. Consequently, the reporting time is condensed in the proposed scheme. The sensing performance of the proposed CSS (PCSS) for all possible fusion rules is reviewed. Analytical expressions for probability of false alarm, probability of detection and throughput are derived for PCSS. Both simulation results and theoretical analyses validate that PCSS improves throughput significantly when compared with CSS.
\end{abstract}

Keywords: CR, CSS, PCSS, ED, PU, SU, Sensing time, Reporting time, Throughput.

\section{Introduction}

As stated by the Federal Communications Commission survey, most of the licensed spectrum is un-utilized $[1,2]$. FCC recognized $\mathrm{CR}$ as the candidate for resourceful spectrum utilization. The CR network comprises spectrum sensing, spectrum management, spectrum sharing and spectrum mobility [3]. CR network authorizations SU (unlicensed spectrum user) to utilize the licensed spectrum band dedicated to the PU when the spectrum band is provisionally not being used. Spectrum sensing methods can be categorized as synchronous detection and non-synchronous detection. The methods of synchronous detection are matched filter detection $[4,5]$ and cyclostationary feature detection [6-8], whereas energy detection is a non-synchronous detection method. The practical method for spectrum sensing is energy detection [911] which functions as a blind detection $[12,13]$. Sensing un-utilized spectrum in wireless environment $[14,15]$ is one of the significant challenges in CR network. In order to enhance spectrum utilization, CSS which takes advantage of special diversity is recommended for wireless environment [16-19]. However, reporting time is a drawback of CSS as it requires local sensing reports of each SU over a shared control channel, which is band limited. From hardware point of view, one should choose parallel reporting [20] but this process increases the computational complexity. Therefore, serial reporting is more enviable [21]. Authors in [22] present double threshold scheme to enhance throughput in CR network. Authors in [23] optimize the sensing duration in order to accomplish maximum throughput and consider the throughput of secondary network only instead of entire CR 
network. Authors in [24] present adaptive sensing duration and detection threshold methods to enhance the throughput in CR network. Similarly, several methods have been suggested to enhance throughput in CSS via adapting system time parameters such as sensing and reporting time. In CSS, one FC is used to collect sensing results from all $\mathrm{k}$ SUs, hence the total reporting time is $\mathrm{k}$ times the reporting time of each SU. However, in PCSS, two FCs are employed for collecting sensing results from two individual groups and final decision is taken by the third FC. As a result, the reporting time is reduced henceforth the data transmission time rises, which is the main advantage in PCSS when compared with CSS. Further, mathematical expressions for throughput are derived for the proposed system employing OR OR OR fusion rule, which yields best sensing performance.

The remainder of this paper is organized as follows. In Section 2, spectrum sensing is reviewed. In section 3, cooperative spectrum sensing is discussed. In Section 4, proposed scheme is presented. Section 5 presents throughput analysis of the proposed scheme. The results are presented in Section 6, and finally conclusions are drawn in Section 7.

\section{Spectrum sensing preliminaries}

Consider a simple CR as illustrated in Fig.1. If PU is absent (channel is idle), the SU transmits the data in licensed spectrum band of PU. If the PU is present (channel is occupied), the sensing is being continued for every predetermined time slot until SU finds unutilized licensed band. The energy $(\mathcal{E})$ of the received signal $y(t)$ is evaluated and compared with threshold $\left(\lambda^{\mathrm{ED}}\right)$ of the energy detector (ED). If $\mathcal{E}<\lambda^{\mathrm{ED}}$, the channel is assumed to be idle, on the contrary, the channel is assumed to be occupied.

Analytically, signal detection can be formalized as a hypothesis test [25]

When the channel is idle:

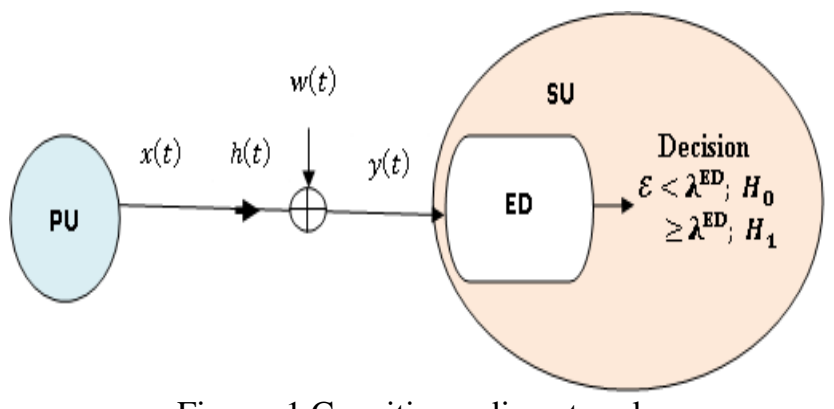

Figure. 1 Cognitive radio network

$$
\mathcal{H}_{0}: y(t)=w(t)
$$

when the channel is occupied by PU:

$$
\mathcal{H}_{1}: y(t)=h(t) x(t)+w(t)
$$

where

$y(t)=$ signal received at the $\mathrm{SU}$

$h(t)=$ gain of sensing channel between SU and PU

$w(t)=$ additive white Gaussian noise (AWGN)

$x(t)=$ signal transmitted from PU

In a binary hypothesis test, if $\mathcal{H}_{1}$ is confessed when $\mathcal{H}_{0}$ is true, it is called type I error and the probability of making this error is called the probability of false alarm $\left(\mathcal{P}_{f}\right)$. If $\mathcal{H}_{0}$ is confessed when $\mathcal{H}_{1}$ is true, it is called type II error and the probability of making this error is called the probability of missed detection $\left(\mathcal{P}_{m}\right)$. In AWGN channel, $\mathcal{P}_{f}$ and $\mathcal{P}_{m}$ can be characterized, respectively, as [14]

$$
\begin{gathered}
\mathcal{P}_{f}=\frac{\Gamma\left(u \frac{\lambda^{\mathrm{ED}}}{2}\right)}{\Gamma(u)} \\
\mathcal{P}_{m}=1-\mathcal{P}_{d}=1-\mathcal{Q}_{u}\left(\sqrt{2 \gamma}, \sqrt{\lambda^{\mathrm{ED}}}\right)
\end{gathered}
$$

where,

$u=$ time-bandwidth product of the ED

$\lambda^{\mathrm{ED}}=$ threshold of the ED

$\gamma=$ SNR of PU

$\mathcal{P}_{d}=$ probability of detection (Probability that the detector states the presence of PU, when the PU is actually present)

$\Gamma(\cdot, \cdot)=$ incomplete gamma function which is specified by

$$
\Gamma(a, b)=\int_{b}^{\infty} t^{a-1} e^{-t} \cdot d t
$$

$\Gamma(\cdot, 0)=\Gamma(\cdot),=$ complete gamma function $\mathcal{Q}_{u}(\cdot, \cdot)=u$ th order generalized Marcum Q function, which is specified by

$$
\mathcal{Q}_{u}(a, b)=\frac{1}{a^{u-1}} \int_{b}^{\infty} t^{u} e^{-\frac{\left(t^{2}+a^{2}\right)}{2}} I_{u-1}(a t) d t
$$

where, $I_{u-1}(\cdot)$ is the $(u-1)$ th order modified Bessel function.

Probability of missed detection versus probability of false alarm of the simple CR for $\gamma=$ $0 \mathrm{~dB}, 10 \mathrm{~dB}, 20 \mathrm{~dB}$, is shown in Fig.2. These curves are called complimentary receiver operating 
characteristics (ROC). In simulation, we consider $u$ $=5$.

It is perceived that as $\gamma$ decreases, the ROC curves shift upward. In other words, the sensing performance gets worse when $\gamma$ decreases. In such scenario, the cooperative decision of multiple CRs can improve the sensing performance as discussed in the subsequent section.

\section{Cooperative spectrum sensing based $\mathbf{C R}$}

Consider a CSS based CR consists of a PU, $\mathrm{k}$ number of cooperative SUs and FC. Sensing is performed by each SU and these results are reported to the FC sequentially. The sensing performance of the CSS depends on number of SUs that are considered $(l)$ at FC to take final decision. The $l$ is called decision threshold. The false alarm probability $\left(Q_{f}{ }^{C S S}\right)$ and detection probability $\left(Q_{d}{ }^{C S S}\right)$ of CSS are delineated as [25-26]

$$
\begin{aligned}
& \mathcal{Q}_{f}{ }^{C S S}=\sum_{i=l}^{k} k c_{i} \mathcal{P}_{f}{ }^{i}\left(1-\mathcal{P}_{f}\right)^{k-i} \\
& \mathcal{Q}_{d}{ }^{C S S}=\sum_{i=l}^{k} k c_{i} \mathcal{P}_{d}^{i}\left(1-\mathcal{P}_{d}\right)^{k-i}
\end{aligned}
$$

Fig. 3 shows the spectrum sensing performance for $\mathrm{k}=10$ and $\gamma=10 \mathrm{~dB}$ with $l=1$ (OR fusion rule), $l=k$ (AND fusion rule) and $l=\frac{k}{2}$ (MAJORITY fusion rule). From Fig.2 and Fig.3, it is apparent that the OR fusion rule gives better sensing performance than that of other fusion rules. Further, when compared with ROC of simple CR (Fig.2), the performance of CSS is increased for the same SNR. The spectrum sensing performance of OR fusion rule for different values of $\mathrm{k}$ is depicted in Fig.4.

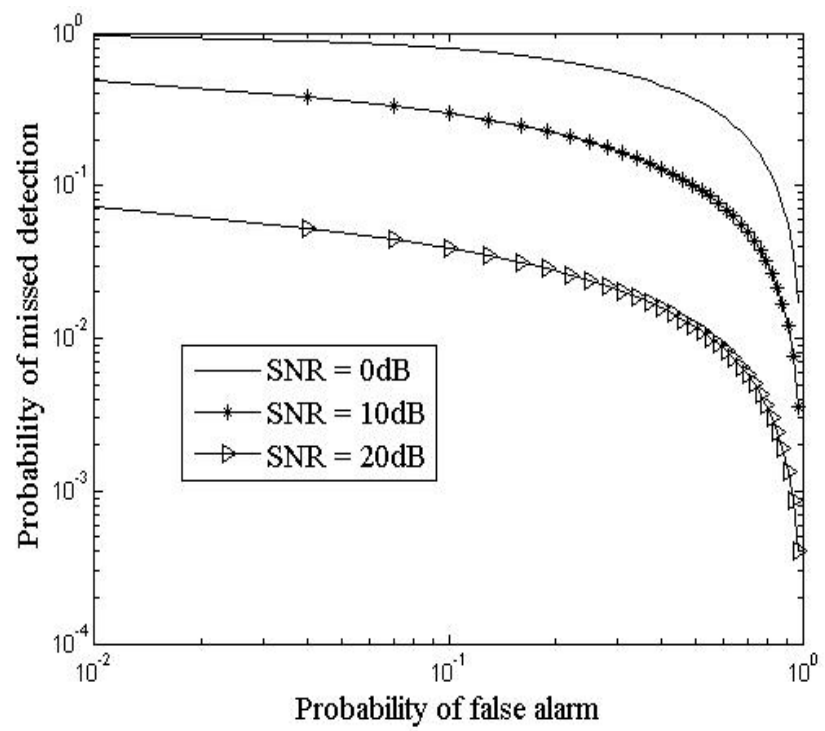

Figure.2 Spectrum sensing performance for simple CR

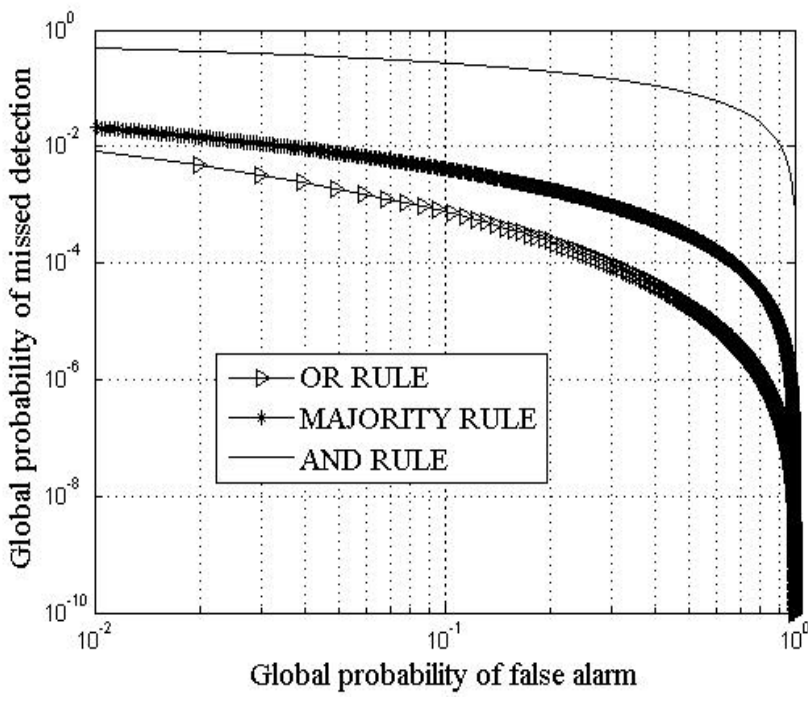

Figure. 3 Spectrum sensing performance in CSS for $\mathrm{k}=10$ with different fusion rules

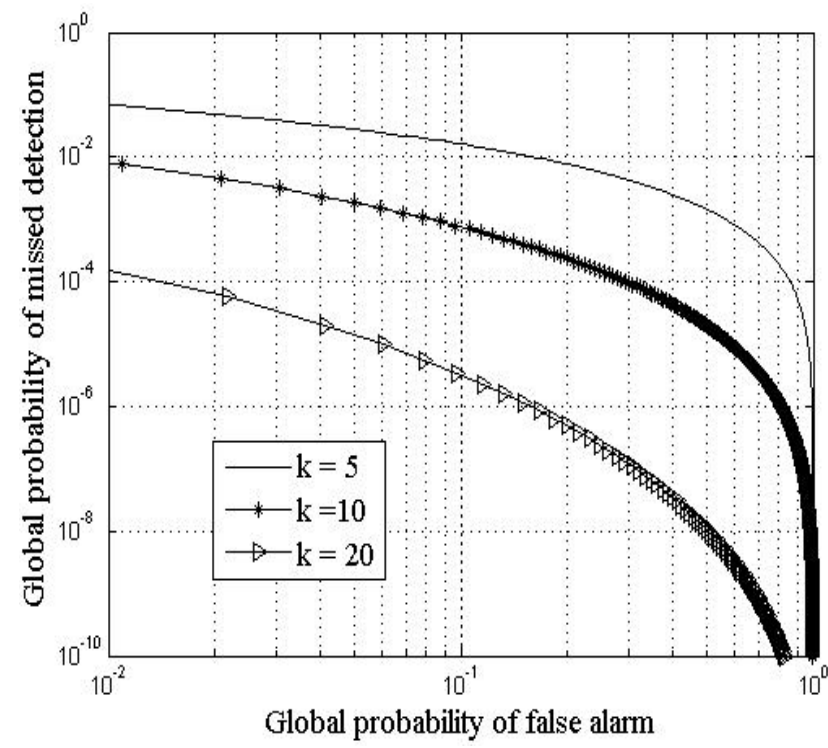

Figure.4 Spectrum sensing performance in CSS for different values of $\mathrm{k}$ with $\mathrm{OR}$ fusion rule

It can be seen that higher the value of $\mathrm{k}$, higher the sensing performance.

\section{Proposed cooperative spectrum sensing scheme}

The proposed cooperative spectrum sensing based CR consists of PU, k SUs, where $\mathrm{k}$ is even, and three FCs as shown in Fig.5. The SUs are divided into two groups with $\frac{k}{2} \mathrm{SUs}$ in each. The SUs in group-I and group-II report their sensing results to FC1 and FC2, respectively. FC1 and FC2 forward their sensing results to FC3, where the decision on the occupancy of the PU is finalized. 


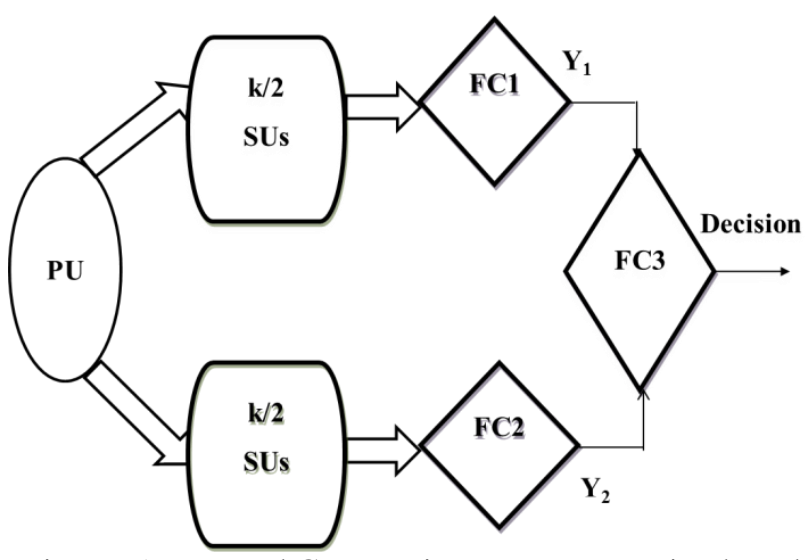

Figure. 5 Proposed Cooperative spectrum sensing based CR

Let $Y_{1}$ and $Y_{2}$ be the fusing results obtained from all 1 bit decisions at $\mathrm{FC} 1$ and $\mathrm{FC} 2$, respectively. Then

$$
Y_{1}=Y_{2}=\sum_{i=1}^{k / 2} S_{i}
$$

where, $S_{i} \in\{0$ (PU is present), 1 (PU is absent) $\}$ is the local decision of ith SU.

Let $m_{1}, m_{2}$ and $n$ be the thresholds at FC1, FC2 and $\mathrm{FC} 3$ respectively. Then the inferences drawn by the FC1, FC2 and FC3 that the channel is accommodated by the PU or idle can be formulized as

At FC1 $: \mathcal{H}_{0}: Y_{1}<m_{1}$ and $\mathcal{H}_{1}: Y_{1} \geq, m_{1}$

At FC2 $: \mathcal{H}_{0}: Y_{2}<m_{2}$ and $\mathcal{H}_{1}: Y_{2} \geq m_{2}$

At FC3: $\mathcal{H}_{0}: Y_{1}+Y_{2}<n$ and $\mathcal{H}_{1}: Y_{1}+Y_{2} \geq n$

False alarm probability and detection probability at $\mathrm{FC} 1$ can be written as, respectively

$$
\begin{aligned}
& {\widetilde{Q_{f}}}^{1}=\sum_{i=m_{1}}^{k / 2} \frac{k}{2} c_{i} \mathcal{P}_{f}^{i}\left(1-\mathcal{P}_{f}\right)^{\frac{k}{2}-i} \\
& {\widetilde{Q_{d}}}^{1}=\sum_{i=m_{1}}^{k / 2} \frac{k}{2} c_{i} \mathcal{P}_{d}^{i}\left(1-\mathcal{P}_{d}\right)^{\frac{k}{2}-i}
\end{aligned}
$$

False alarm probability and detection probability at FC2 can be written as, respectively

$$
\begin{aligned}
& {\widetilde{Q_{f}}}^{2}=\sum_{i=m_{2}}^{k / 2} \frac{k}{2} c_{i} \mathcal{P}_{f}^{i}\left(1-\mathcal{P}_{f}\right)^{\frac{k}{2}-i} \\
& {\widetilde{Q_{d}}}^{2}=\sum_{i=m_{2}}^{k / 2} \frac{k}{2} c_{i} \mathcal{P}_{d}^{i}\left(1-\mathcal{P}_{d}\right)^{\frac{k}{2}-i}
\end{aligned}
$$

All possible fusion rules and their sensing performance of the proposed system are listed in Table 1and Fig.6, respectively.
Table 1. Fusion rules in PCSS

$$
\left(m_{1}, m_{2}, n\right) \quad \text { Fusion rule }
$$

$(1,1,1) \quad$ OR OR OR

$\left(\frac{k}{2}, \frac{k}{2}, 1\right) \quad$ AND AND OR

$(1,1,2) \quad$ OR OR AND

$\left(\frac{k}{2}, \frac{k}{2}, 2\right) \quad$ AND AND AND

$\left(\frac{k}{2}, 1,1\right) \quad$ AND OR OR

$\left(\frac{k}{2}, 1,2\right) \quad$ AND OR AND

$\left(\frac{k}{4}, \frac{k}{4}, 1\right) \quad$ MAJORITY MAJORITY OR

$\left(\frac{k}{4}, \frac{k}{4}, 2\right) \quad$ MAJORITY MAJORITY AND

$\left(\frac{k}{4}, 1,2\right) \quad$ MAJORITY OR AND

$\left(\frac{k}{4}, 1,1\right) \quad$ MAJORITY OR OR

$\left(\frac{k}{4}, \frac{k}{2}, 1\right) \quad$ MAJORITY AND OR

$\left(\frac{k}{4}, \frac{k}{2}, 2\right) \quad$ MAJORITY AND AND

According to Fig.6, among all 12 possible fusion rules, OR OR OR fusion rule shows best sensing performance. Hence, to keep the same sensing performance in PCSS, we consider OR OR OR fusion rule in our proposed work.

Since OR OR fusion rules are employed at FC1 and FC2, let us assume $m_{1}=m_{2}=m$. Therefore, we get

$$
\begin{aligned}
& {\widetilde{Q_{f}}}^{1}={\widetilde{Q_{f}}}^{2}=\widetilde{Q_{f}} \\
& {\widetilde{Q_{d}}}^{1}={\widetilde{Q_{d}}}^{2}=\widetilde{Q_{d}}
\end{aligned}
$$

where,

$$
\begin{aligned}
\widetilde{Q_{f}} & =1-\left(1-\mathcal{P}_{f}\right)^{\frac{k}{2}} \\
\widetilde{Q_{d}} & =1-\left(1-\mathcal{P}_{d}\right)^{\frac{k}{2}}
\end{aligned}
$$




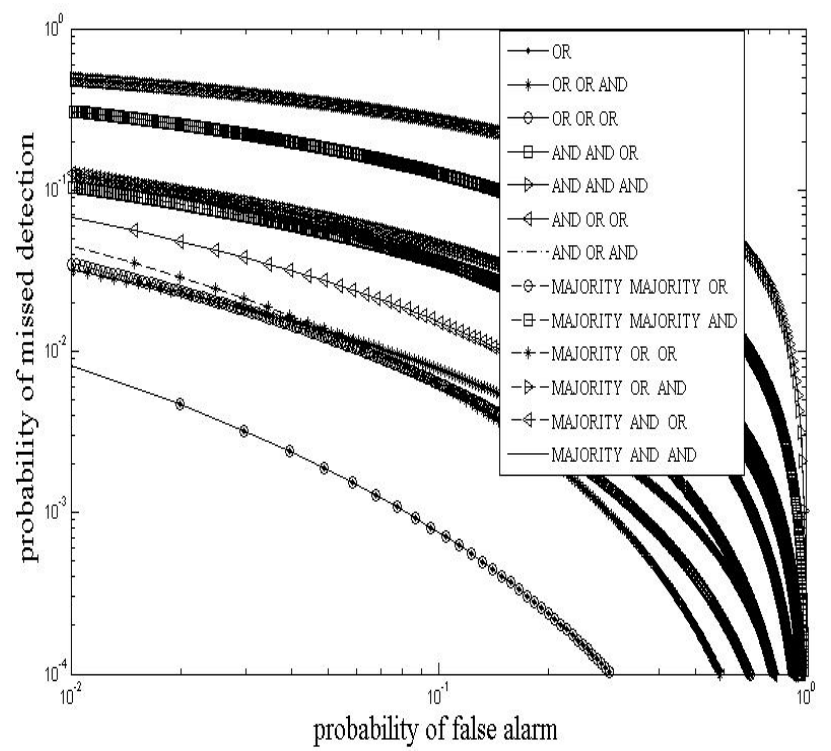

Figure. 6 Spectrum sensing performance in PCSS with all possible fusion rules

Further, the probabilities at FC3 for $\mathrm{n}$ out of 2 fusion rule can be written as

$$
\begin{aligned}
& \mathcal{Q}_{f}{ }^{P C S S}=\sum_{i=n}^{2} 2 c_{i}{\widetilde{Q_{f}}}^{i}\left(1-\widetilde{Q_{f}}\right)^{2-i} \\
& \mathcal{Q}_{d}{ }^{P C S S}=\sum_{i=n}^{2} 2 c_{i}{\widetilde{Q_{d}}}^{i}\left(1-\widetilde{Q_{d}}\right)^{2-i}
\end{aligned}
$$

Since OR fusion rule is employed at FC3, Eqs. (18) and (19) can be simplified as

$$
\begin{aligned}
& \mathcal{Q}_{f}{ }^{\text {PCSS }}=1-\left(1-\widetilde{Q_{f}}\right)^{2} \\
& \mathcal{Q}_{d}{ }^{\text {PCSS }}=1-\left(1-\widetilde{Q_{d}}\right)^{2}
\end{aligned}
$$

\section{Throughput analysis of PCSS}

In conventional communication systems, entire time frame is utilized for data transmission. But in $\mathrm{CR}$, some portion of time frame is devoted for sensing and hence data transmission time is reduced. Due to reporting time in CSS based CR, the data transmission is further reduced. Eventually, throughput (bits/second) of CSS based CR is lower than that of simple CR. In this work we present a method to reduce the reporting time so as to increase the data transmission time, consequently, the throughput increases.

The conventional frame structure that is employed in CSS is presented in Fig.7. From Fig.7, we can express

$$
T_{R}=T_{F}-T_{S}-T_{D}
$$

Obviously, for a given time frame $\left(T_{F}\right)$ and sensing time $\left(T_{S}\right)$ the reporting time $\left(T_{R}\right)$ is inversely proportional to data transmission time $\left(T_{D}\right)$.

Fig.8 presents frame structure that is employed in PCSS. According to the frame structure of the PCSS, the reporting time is the sum of time required to report the decision of $\frac{k}{2} \mathrm{SUs}$ in group-I to FC1, time required to report the decision of $\frac{k}{2} \mathrm{SUs}$ in group-II to $\mathrm{FC} 2$ and time required to forward the decisions of FC1 and FC2 to FC3.

In above frame structure,

Reporting time of ith SU $=t_{r, i}$

Reporting time of FC1 $\quad=t_{f c 1}$

Reporting time of FC2 $\quad=t_{f c 2}$

Also,

$t_{r, 1}=t_{r, 2}=\cdots=t_{r, \frac{k}{2}}=t_{f c 1}=t_{f c 2}=t_{r}$

Reporting time in PCSS can be written as

$$
T_{R}=\left(\frac{k}{2}+2\right) \cdot t_{r}
$$

From Fig.7, we can easily derived that the reporting time in CSS as

$$
T_{R}=k \cdot t_{r}
$$

The amount of reporting time saved $\left(\Delta T_{R}\right)$ can be expressed as

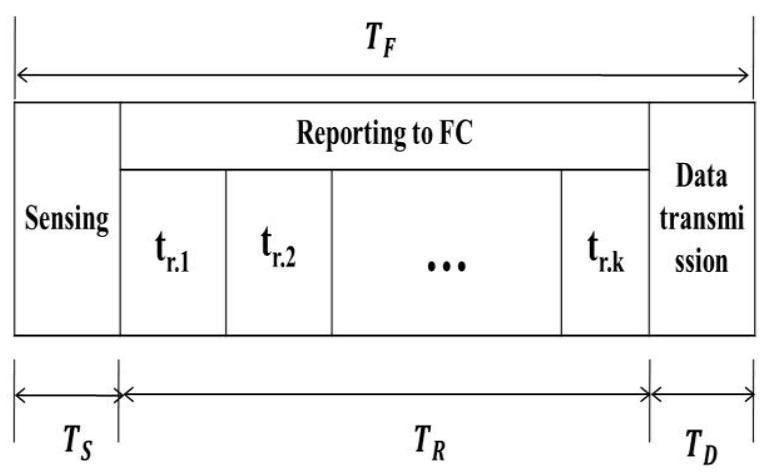

Figure. 7 Conventional frame structure

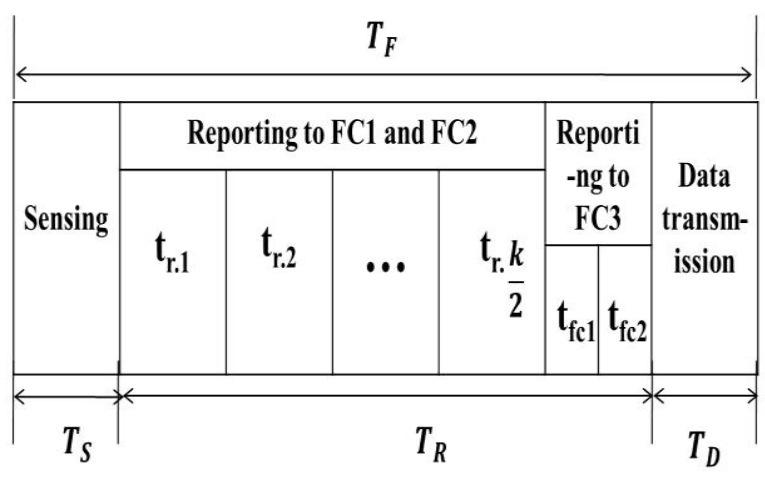

Figure. 8 Frame structure in PCSS 
Table 2. Activities of PU and SU

\begin{tabular}{cccc}
\hline Scenario & $\begin{array}{c}\text { PU } \\
\text { status }\end{array}$ & $\begin{array}{c}\text { Decision } \\
\text { at CR }\end{array}$ & $\begin{array}{c}\text { Channel } \\
\text { occupancy }\end{array}$ \\
\hline 1 & Present & Present & only by PU \\
2 & Present & Absent & $\begin{array}{c}\text { simultaneously } \\
\text { by PU and SU }\end{array}$ \\
3 & Absent & Absent & $\begin{array}{c}\text { only by SU } \\
\text { neither by PU } \\
\text { nor by SU }\end{array}$ \\
\hline
\end{tabular}

$\Delta T_{R}=$ Reporting time in CSS - Reporting time in PCSS

From Eq. (23) and Eq. (24), we get

$$
\Delta T_{R}=\left(\frac{\mathrm{k}}{2}-2\right) \cdot t_{\mathrm{r}}
$$

There are four states between the actions of SU and PU as illustrated in Table 2.

For evaluating throughput, we consider the scenario 3, in which SU transmits data in the absence of PU. Then the throughput for scenario 3 is given as [23]

$$
\mathcal{T}_{s u}=\frac{T_{F}-T_{S}-T_{R}}{T_{F}} \log _{2}\left(1+\gamma_{s u}\right)
$$

where,

$\gamma_{s u}=\mathrm{SNR}$ of SU

Therefore, average achievable throughput of the PCSS is given as

$$
\mathcal{T}_{\text {average }}=\mathcal{P}\left(\mathcal{H}_{0}\right)\left(1-\mathcal{Q}_{f}{ }^{\text {PCSS }}\right) \mathcal{T}_{\text {su }}
$$

where,

$\mathcal{P}\left(\mathcal{H}_{0}\right)=$ Probability that the channel is idle

Substituting Eq. (21) in Eq. (22), we get

$$
\mathcal{T}_{\text {average }}=f\left(T_{S}\right)-T_{R} g\left(T_{S}\right)
$$

where,

$$
\begin{aligned}
& f\left(T_{S}\right)= \\
& \frac{T_{F}-T_{S}}{T_{F}} \mathcal{P}\left(\mathcal{H}_{0}\right)\left(1-\mathcal{Q}_{f}{ }^{P C S S}\right) \log _{2}\left(1+\gamma_{s u}\right) \\
& g\left(T_{S}\right)= \\
& \frac{1}{T_{F}} \mathcal{P}\left(\mathcal{H}_{0}\right)\left(1-\mathcal{Q}_{f}{ }^{P C S S}\right) \log _{2}\left(1+\gamma_{s u}\right)
\end{aligned}
$$

\section{Results and discussion}

The ROC curves of CSS offered in [25] and PCSS are portrayed in Fig.3 and Fig.6, respectively. It is perceived that error performance in CSS with OR fusion rule is similar to that of PCSS with OR OR OR fusion rule. From Eq.(24) and Eq.(25), it is clear that the reporting time in PCSS is low when compared with CSS. Further, from Eq.(26) and Eq.(27), throughput is inversely proportional to reporting time hence throughput of PCSS is more when compared with CSS. The simulation results of throughput in CSS and PCSS are presented in this section.

In our simulation, we consider bandwidth of the PU signal is $2 \mathrm{MHz}$, modulation is BPSK, sampling frequency is the same as that of the PU signal, $T_{F}=$ $20 \mathrm{~ms}, t_{r}=0.01 \mathrm{~ms}$, SNR of PU signal $=5 \mathrm{~dB}, \mathrm{SNR}$ of $\mathrm{SU}=10 \mathrm{~dB}$ and $\mathcal{P}\left(\mathcal{H}_{0}\right)=0.5$. We also adapted OR fusion rule in CSS and OR OR OR fusion rule in PCSS while these fusion rules bestow the best sensing performance as proved in section 4 .

Fig.9 presents average achievable throughput versus sensing time for CSS that employs conventional frame structure (Fig.7) and PCSS that employs proposed frame structure (Fig.8) for $\mathrm{k}=50$, 100, 200.

From Fig.9, it is perceived that there is an optimal sensing time to attain maximum throughput for every value of $\mathrm{k}$ for both PCSS and CSS. It can also be seen that the throughput performance of PCSS at every value of sensing time is higher than that of CSS. Further, the gain in average achievable throughput increases with $\mathrm{k}$.

Fig.10 presents average achievable throughput versus number of SUs for CSS that employs conventional frame structure (Fig.7) and PCSS that employs proposed frame structure (Fig.8) for $T_{S}=$ $7 \mathrm{~ms}$.

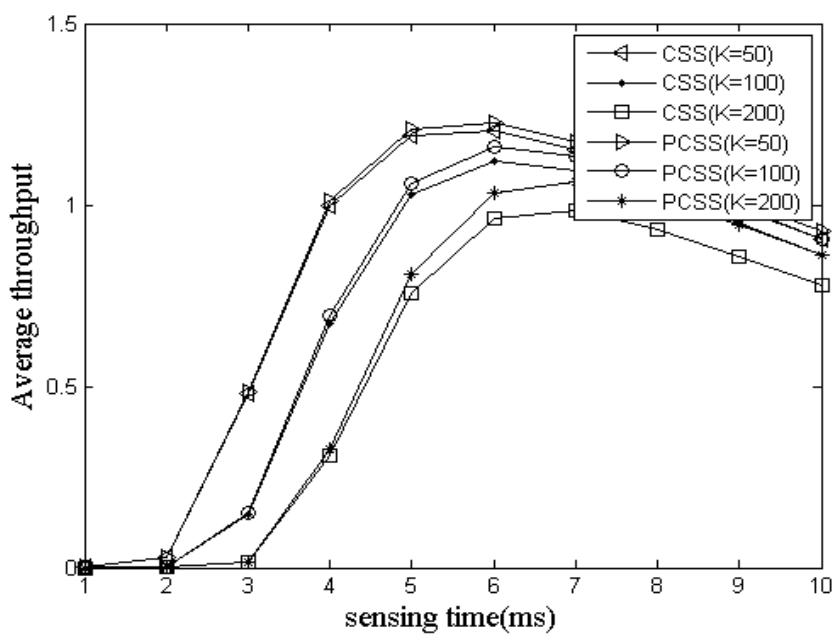

Figure. 9 Average throughput versus sensing time 


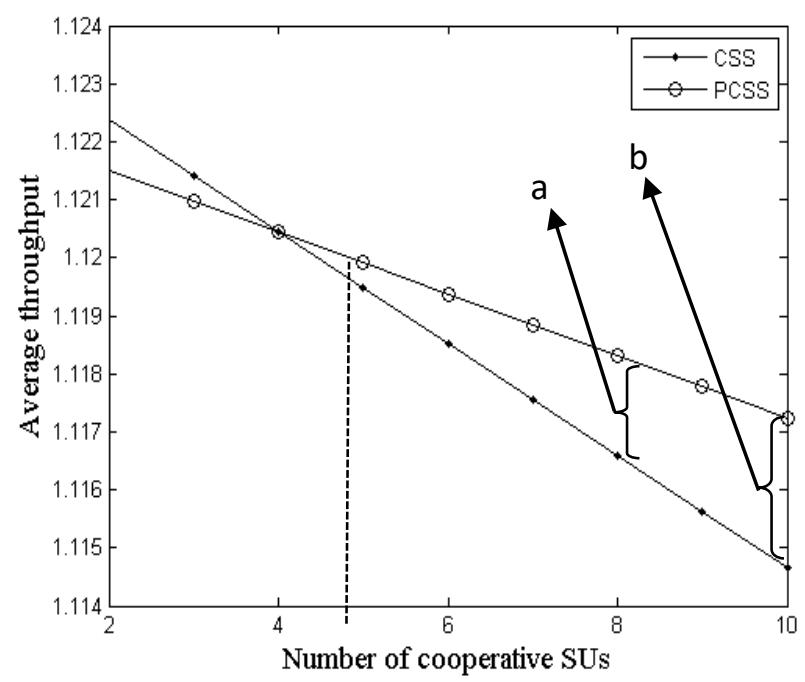

Figure. 10 Average throughput versus number of cooperative SUs

The performance of the PCSS is explained as under

- Case 1: $\mathrm{k}<4\left(\Delta T_{R}<0\right)$; average achievable throughput of PCSS is lower than that of CSS.

- Case 2: $\mathrm{k}=4\left(\Delta T_{R}=0\right)$; average achievable throughput of PCSS is similar to that of CSS.

- Case 3: $k>4$ (where $\mathrm{k}$ is even) $\left(\Delta T_{R}>0\right)$; average achievable throughput of PCSS is higher than that of CSS. For instance, when $\mathrm{k}$ $=8$, the average achievable throughput of CSS and PCSS is 1.116 and 1.118, respectively. Therefore, the gain in average achievable throughput is $0.002(=\mathrm{a})$. Further average achievable throughput degradation with $\mathrm{k}$ in PCSS is lower than that of CSS. For example, when $\mathrm{k}$ increases to 10 , the gain in average achievable throughput increases to $0.003(=b)$ in PCSS.

\section{Conclusions}

In this paper, we proposed a frame structure that reduces the reporting time, when compared with conventional frame structure. As a result the average achievable throughput of PCSS is improved significantly. The sensing performance of the proposed CSS based CR is plotted for all 12 possible fusion rules and it has been found that OR OR OR fusion rule presents best sensing performance. Probability of false alarm, probability of missed detection and average throughput of the PCSS are derived with OR OR OR fusion rule. In PCSS, the amount of reporting time saved when compared with CSS that employs conventional frame structure is $\left(\frac{\mathrm{k}}{2}-2\right) \cdot t_{\mathrm{r}}$. Eventually, for $k>4$ (where $\mathrm{k}$ is even), the degradation in throughput performance with $\mathrm{k}$ of PCSS is lower than that of CSS at constant number of SUs. Further, this degradation in throughput performance decreases as $\mathrm{k}$ increases. For instance, when $\mathrm{k}$ increases from 8 to 10 , the gain in average achievable throughput is increased from 0.002 to 0.003 . Adaptive algorithms may be fused in the proposed system for further throughput enhancement.

\section{References}

[1] Communications Commission, "Spectrum policy task force report" U.K Government, 2002.

[2] Federal Communications Commission, "Notice of Proposed Rulemaking on Cognitive Radio", FCC Washington, 2003.

[3] I. F. Akyildiz, W. Lee, M. C. Vuran, and S. Mohanty, "Next generation/dynamic spectrum access/cognitive radio wireless networks: A survey", Journal of Computer and Telecommunication Networking, Vol.50, No.13, pp. 2127-2159, 2009.

[4] Q. Lv and F. Gao, "Matched filter based spectrum sensing and power level recognition with multiple antennas", Proc. of IEEE China Summit and International Conference on Signal Information, Chengdu, pp. 305-309, 2016.

[5] D. Bhargavi and C. R. Murthy, "Performance comparison of energy, matched-filter and cyclostationarity-based spectrum sensing", Proc. of IEEE 11th International Workshop on Signal Processing Advances in Wireless Communications, Marrakech, Morocco, pp. 1-5, 2010.

[6] A. Patel, S. Biswas, and A. K. Jaganatham, "Multiple Beacon based robust cooperative spectrum sensing in MIMO cognitive radio networks", Proc. of IEEE 78th Vehicular Technology Conference, Las Vegas, NV, USA, pp. 1-5, 2013.

[7] P. Urriza, E. Rebeiz, and D. Cabric, "Multiple antenna cyclostationary spectrum sensing based on the cyclic correlation significance Test", IEEE J. Selected Areas in Communications, Vol.31, No.11, pp. 2185-2195, 2013.

[8] J. Lunden, V. Koivunen, and A. Huttunen, "Collaborative cyclostationary spectrum sensing for cognitive radio systems", IEEE Transactions on Signal Processing, Vol.7, No.11, pp. 4182-4195, 2009.

[9] A. S. B. Kozal, M. Merabti, and F. Bouhafs, "An improved energy detection scheme for cognitive radio networks in low SNR region", 
Proc. of IEEE Symposium on Computers and Communications, pp. 684-689, 2012.

[10] S. Tapattu, C. Tellambura, J. Hai, and N. Rajatheva, "Unified analysis of low-SNR energy detection and threshold selection", IEEE Transactions on Vehicular Technology, Vol.64, No.11, pp. 5006-5019, 2014.

[11] C. Vladeanu, C.V. Nastase, and A. Martian, "Energy detection algorithm for spectrum sensing using three consecutive sensing events", IEEE Wireless Communications Letters, Vol.5, No.3, pp. 284-287, 2016.

[12] H. Urkowitz, "Energy detection of unknown deterministic signals", Proc. of IEEE, Vol.55, No.4, pp. 523-531, 1967.

[13] W. Yin, P. Ren, Z. Su, M. A, and Ruijuan, “A multiple antenna spectrum sensing scheme based on space and time diversity in cognitive radios", IEICE Transactions on Communications, Vol.94, No.5, pp. 1254-1264, 2011.

[14] F. F. Digham, M. S. Alouini, and M. K. Simon, "On the energy detection of unknown signals over fading channels", IEEE Transactions on Communications, Vol.55, No.1, pp. 21-24, 2007.

[15] G. Baldini, R. Giuliani, D. Capriglone, and K. Sithamparanathan, : Foundation of cognitive radio systems, INTECH Publisher, Europe, 2012.

[16] S. K. Syed-Yusof, K. M. Khairul Rashid, N. M. Abdul Latiff, N. Fisal, M. A. Sarijari, R. A. Radhid, and N. Ramli, "TDMA-based cooperative sensing using SDR platform for cognitive radio", IEEE $18^{\text {th }}$ Asia-Pacific conference on communications, Jeju Island, South Korea, pp. 278-283, 2012.

[17] J. Ma and Y. Li, "Soft combination and detection for cooperative spectrum sensing in cognitive radio networks", Global Telecommunications Conference, IEEE, Washington, DC, USA, pp. 3139-3143, 2007.

[18] A. Sendonaris, E. Erkip, and B. Aazhang, "User cooperation diversity. Part 1. System description", IEEE Transactions on Communications, Vol.51, No.11, pp. 19271938, 2003.

[19] A. Sendonaris, E. Erkip, and B. Aazhang, "User cooperation diversity. Part II. Implementation aspects and performance analysis", IEEE Transactions on Communications, Vol.51, No.11, pp. 1939-1948, 2003.

[20] S. Zhang, T. Wu, and V. K. N. Lau, "A lowoverhead energy detection based cooperative sensing protocol for cognitive radio systems",
IEEE Transactions on Wireless Communications, Vol.8, No.11, pp. 5575-5581, 2009.

[21] G. Feng, Y. Wei, L. Wei, C. Wenqing, and W. Shu, "Pipelined cooperative spectrum sensing in cognitive radio networks", IEEE Wireless Communications and Networking Conference, Budapest, Hungary, pp. 1-5, 2009.

[22] G. Verma and O. P. Sahu, "Throughput Maximization of Cognitive Radio Under the Optimization of Sensing Duration", Wireless Personal Communications, DOI 10.1007/s11277-017-4564-x, 2017.

[23] C. L. Ying, Z. Yonghong, C. Y. P. Edward, and T. H. Anh, "Sensing Throughput Trade-off for Cognitive Radio Networks", IEEE Transactions on Wireless Communications, Vol.7, No.4, pp. 1326-1337, 2008.

[24] W. B. Tae, S. K. Jun, and C. J. Bang, "Adaptive Spectrum Sensing for Throughput Maximization of Cognitive Radio Networks in Fading Channels", Journal of Information and Communication Convergence Engineering, Vol.9, No.3, pp. 251-255, 2011.

[25] K. B. Letaief, and W. Zhang, "Cooperative Communications for Cognitive Radio Networks", In: Proc. of IEEE, Vol.97, No.5, pp. 878-893, 2009.

[26] H. Hang, Z. Hang, Y. Hong, Y. Chen, and J. Javad, "Energy-efficient design of channel sensing in cognitive radio networks", Computers and Electrical Engineering, Vol.42, No.4, pp. 207-220, 2015. 